\title{
Leptogenesis in $\mathrm{SO}(10)$
}

\author{
Chee Sheng Fong, ${ }^{a}$ Davide Meloni, ${ }^{b}$ Aurora Meroni ${ }^{c}$ and Enrico Nardi ${ }^{d}$ \\ ${ }^{a}$ Instituto de Física, Universidade de São Paulo, \\ C.P. 66.318, 05315-970 São Paulo, Brazil \\ ${ }^{b}$ Dipartimento di Matematica e Fisica, \\ Via della Vasca Navale 84, 00146 Roma \\ ${ }^{c} C P^{3}$-Origins $\&$ the Danish Institute for Advanced Study Danish IAS, \\ Univ. of Southern Denmark, Campusvej 55, DK-5230 Odense, Denmark \\ ${ }^{d}$ INFN - Laboratori Nazionali di Frascati, \\ C.P. 13, 100044 Frascati, Italy \\ E-mail: fong@if.usp.br, meloni@fis.uniroma3.it, \\ meroni@cp3.dias.sdu.dk, enrico.nardi@lnf.infn.it
}

AbStract: We consider SO(10) Grand Unified Theories (GUTs) with vacuum expectation values (vevs) for fermion masses in the $\mathbf{1 0}+\overline{\mathbf{1 2 6}}$ representation. We show that the baryon asymmetry generated via leptogenesis is completely determined in terms of measured low energy observables and of one single high energy parameter related to the ratio of the $\mathbf{1 0}$ and $\overline{\mathbf{1 2 6}} \mathrm{SU}(2)$ doublet vevs. We identify new decay channels for the heavy Majorana neutrinos into $\mathrm{SU}(2)$ singlet leptons $e^{c}$ which can sizeably affect the size of the resulting baryon asymmetry. We describe how to equip $\mathrm{SO}(10)$ fits to low energy data with the additional constraint of successful leptogenesis, and we apply this procedure to the fits carried out in ref. [1]. We show that a baryon asymmetry in perfect agreement with observations is obtained.

KeYwords: Beyond Standard Model, GUT

ARXIV EPRINT: 1412.4776 


\section{Contents}

1 Introduction 1

2 Yukawa couplings 3

3 Leptogenesis Lagrangian and CP asymmetries 5

$\begin{array}{lll}3.1 & \mathrm{CP} \text { violating asymmetries } & 6\end{array}$

3.2 Boltzmann Equations 8

4 Relating leptogenesis parameters to observables 10

5 Results 12

6 Concluding remarks $\quad 16$

\section{Introduction}

Dark matter, neutrino masses, and the observed Baryon Asymmetry of the Universe (BAU) are all evidences that a theory more fundamental than the Standard Model (SM) must exist. However, so far no other observation has been found to disagree with the SM predictions. In particular, precision electroweak tests, searches for rare flavour changing processes and electric dipole moments, and the results of the first LHC run, have all failed to find new physics, and have not provided hints on how the present theory could be extended. Rather, Nature seems to suggest us that the naturalness paradigm, which has guided the construction of quite appealing extensions of the SM, might need revision: maybe the realm of new physics is way above the electroweak scale, and not around the corner. If this is the case, any information we are able to infer about the structure of the theory at very large energy scales acquires pivotal importance. In this respect, it is unequivocal that the three SM gauge couplings tend to converge while flowing towards high energies, and this strongly suggests that grand unification might be a fundamental feature of the underlying theory, an idea which is also corroborated by the possibility of explaining, within GUT frameworks, electric charge quantization, the absence of SM gauge anomalies, etc. The fact that in the SM gauge coupling unification at a single point does not occur, should not be considered as a problem, but rather as an expected feature, since the three evidences for new physics mentioned above in general require new matter fields below the GUT scale which can affect the running of the couplings and can give rise, for the low energy observer, to a certain amount of aberration rather than to a sharp focus point.

Among the possible GUT groups, $\mathrm{SO}(10)[2-6]$ is particularly interesting for several reasons: it is the smallest group for which the fifteen fermions of one SM generation can 
fit within a single irreducible representation (the spinorial 16), it predicts the existence of one right-handed $(\mathrm{RH})$ neutrino per family which in turn, via the seesaw mechanism, can account for massive light neutrinos, it can allow for non-supersymmetric gauge coupling unification and for a sufficiently long nucleon lifetime [7-12] and, being the group rank five, it can allow for an intermediate scale a few order of magnitude below the GUT scale where the gauge group reduces to rank 4 . Neutrino masses, the mechanism generating the BAU, and possibly also dark matter, might all be related with this scale.

Several connections between $\mathrm{SO}(10)$ high energy parameters and observables can be pinned down by studying the Yukawa sector. Vacuum expectation values (vevs) giving rise to fermion masses must belong to conjugate representations of $\mathbf{1 6} \otimes \mathbf{1 6}=\mathbf{1 0} \oplus \mathbf{1 2 0} \oplus \mathbf{1 2 6}$. In a renormalizable model, the $\overline{\mathbf{1 2 6}}$ is in any case unavoidable since it is the only representation containing a SM singlet, which is needed to implement the seesaw mechanism (otherwise neutrino would have Dirac masses of the order of the charged fermion masses). However, the minimal choice of just one Yukawa coupling is not viable, because it is always possible to rotate the fermionic $\mathbf{1 6}$ to a basis in which the Yukawa matrix is diagonal, with the result that the up- and down-quark masses would be diagonal in the same basis and all the Cabibbo-Kobayashi-Maskawa (CKM) mixings would thus vanish. The possibility of $\mathbf{1 2 0} \oplus \overline{\mathbf{1 2 6}}$ was suggested in [13] but later found, by dedicated numerical analyzes, to be not viable [1, 14]. The option $\mathbf{1 0} \oplus \overline{\mathbf{1 2 6}}$ has been instead found to allow fitting consistently all the low energy data $[1,12,14]$. This can be achieved under the assumption that the neutrino masses are dominated by type I seesaw contributions, and after promoting the fields in the $\mathbf{1 0}$ to complex fields [8]. ${ }^{1}$ Moreover, as it was recently found in [15], in this model both the requirements of gauge coupling unification and of a proton lifetime above the experimental limits can be satisfied.

In conclusion, the results of various studies agree on the fact that the $\mathrm{SO}(10)$ model with scalars in the $\mathbf{1 0} \oplus \overline{\mathbf{1 2 6}}$ (a $\mathbf{4 5}$ is also needed for the correct breaking of the GUT gauge symmetry, however it does not contribute to the SM fermion masses) has so far succeeded in passing a large set of phenomenological tests. In this work we will confront the model with one more test, namely we will study if the $\mathbf{1 0} \oplus \overline{\mathbf{1 2 6}} \mathrm{SO}(10)$ model is able to account for the observed amount of the BAU via the standard mechanism of $\mathrm{CP}$ violating decays of the heavy Majorana neutrinos $N$ and leptogenesis [16-18]. Our main results are that the model is indeed compatible with BAU observations. As a byproduct, we perform a complete disentanglement between the Yukawa coupling matrices and the values of the vevs related to fermion masses (something that cannot be achieved with low energy fits alone) and we also obtain some information about the structure of the intermediate scale particle spectrum. Various studies related to leptogenesis in $\mathrm{SO}(10)$ that rely on different sets of assumptions and/or on variations of the minimal model have appeared in the literature $[12,19-34]$. Here we stick to the minimal SO(10) model constrained only by the condition that the numerical values of the model parameters are such that all the low energy observables are fitted correctly.

\footnotetext{
${ }^{1}$ We refer to $[8,13]$ for details and implications of complexifying the $\mathbf{1 0}$ while forbidding an additional 1616 10* Yukawa coupling.
} 
We start in section 2 by deriving from the $\mathrm{SO}(10)$ Yukawa Lagrangian the couplings of the Majorana neutrinos $N$ to the SM fields, as well as to other $\mathrm{SO}(10)$ fields with intermediate scale masses, which could provide new decay channels for the $N$ 's. In section 3 we compute the leptogenesis $\mathrm{CP}$ asymmetries and we write down the relevant Boltzmann equations. In section 4 we derive the connections between the relevant leptogenesis parameters and quantities that can be fitted from low energy data. In section 5 we apply our results to sets of data points resulting from the fits carried out in ref. [1], and we show that the correct amount of BAU is indeed produced. Finally in section 6 we recap and draw our conclusions.

\section{Yukawa couplings}

Fermions are assigned to three $\mathbf{1 6}_{a}$ spinorial representations where $a=1,2,3$ is a generation index. Scalars are assigned to the (fundamental) vector representation $\mathbf{1 0}_{\mu}$, where $\mu, \nu, \ldots$ are $\mathrm{SO}(10)$ indices, and to the fifth rank antisymmetric tensor $\mathbf{1 2 6}_{\mu \nu \lambda \rho \sigma}$ (satisfying the constraint $\left.\mathbf{1 2 6}_{\mu \nu \lambda \rho \sigma}=i \epsilon_{\mu \nu \lambda \rho \sigma \alpha \beta \gamma \delta \epsilon} \mathbf{1 2 6}_{\alpha \beta \gamma \delta \epsilon}\right)$. Following refs. [35, 36] we write the $\mathrm{SO}(10)$ Yukawa Lagrangian as

$$
\begin{aligned}
\mathcal{L} & =\mathcal{L}_{\mathbf{1 0}}+\mathcal{L}_{\mathbf{1 2 6}}, \\
-\mathcal{L}_{\mathbf{1 0}} & =\tilde{h}_{a b}\left(\mathbf{1 6}_{a}^{T} B C^{-1} \Gamma_{\mu} \mathbf{1 6}_{b}\right) \mathbf{1 0}_{\mu}, \\
-\mathcal{L}_{\mathbf{1 2 6}} & =\frac{1}{5 !} \tilde{f}_{a b}\left(\mathbf{1 6}_{a}^{T} B C^{-1} \Gamma_{\mu} \Gamma_{\nu} \Gamma_{\lambda} \Gamma_{\rho} \Gamma_{\sigma} \mathbf{1 6}_{b}\right) \overline{\mathbf{1 2 6}}_{\mu \nu \lambda \rho \sigma},
\end{aligned}
$$

where $\Gamma_{\mu}$ are the matrices of the ten-dimensional Clifford algebra, $B$ is the charge conjugation matrix for the $\mathrm{SO}(10)$ spinor representation, and $C$ is the charge conjugation matrix for space-time spinors. The Yukawa matrices $\tilde{h}_{a b}$ and $\tilde{f}_{a b}$ are $3 \times 3$ complex symmetric, however by a unitary rotation of the fermion multiplets it is always possible to define a basis in which one of the two matrices is diagonal, with real and positive eigenvalues (and we will later assume the basis in which $\tilde{h}$ is diagonal). Note that such a transformations exhausts the freedom for field redefinition, so that the $3(\tilde{h})+(6+6)(\tilde{f})=15$ remaining Yukawas correspond to physical parameters.

Our goal is to project eq. (2.1) onto multiplets of the $\mathrm{SM}$ gauge group $\mathcal{G}_{\mathrm{SM}}=\mathrm{SU}(2)_{L} \times$ $\mathrm{U}(1) \times \mathrm{SU}(3)$ keeping trace of Clebsch-Gordan coefficients between the various terms. These coefficients can be derived by analyzing the symmetry reduction chain $\mathrm{SO}(10) \supset G_{I} \supset \mathcal{G}_{\mathrm{SM}}$ where the intermediate $G_{I}$ is any (maximal) subgroup of $\mathrm{SO}(10)$ like $\mathcal{G}_{P S}=\mathrm{SU}(2) \times \mathrm{SU}(2) \times$ $\mathrm{SU}(4)$ or $\mathcal{G}_{5}=\mathrm{SU}(5) \times \mathrm{U}(1)$. Clearly, the result does not depend on the particular $G_{I}$ chosen and therefore, even if we have in mind $G_{I}=\mathcal{G}_{P S}$ as the intermediate scale symmetry group, here we will follow the chain $\mathrm{SO}(10) \supset \mathcal{G}_{5} \supset \mathcal{G}_{\mathrm{SM}}$ since the Majorana neutrinos, which are of utmost relevance for our study, are immediately singled out as the $\mathrm{SU}(5) \operatorname{singlets} \mathbf{1}=N$. We need the following $\mathrm{SO}(10) \rightarrow \mathrm{SU}(5)$ branching rules:

$$
\begin{aligned}
\mathbf{1 6} & =\mathbf{1} \oplus \overline{\mathbf{5}} \oplus \mathbf{1 0}, \\
\mathbf{1 0} & =\mathbf{5} \oplus \overline{\mathbf{5}}, \\
\overline{\mathbf{1 2 6}} & =\mathbf{1} \oplus \mathbf{5} \oplus \overline{\mathbf{1 0}} \oplus \mathbf{1 5} \oplus \overline{\mathbf{4 5}} \oplus \mathbf{5 0} .
\end{aligned}
$$


Projecting eq. (2.2) onto the SU(5) multiplets we obtain [36]:

$$
-\mathcal{L}_{\mathbf{1 0}} \rightarrow-\mathcal{L}_{h}=i 2 \sqrt{2} \tilde{h}_{a b}\left[-\mathbf{1}_{a} \overline{\mathbf{5}}_{b i} \mathbf{5}_{H_{u}}^{i}+\mathbf{1 0}_{a}^{i j} \overline{\mathbf{5}}_{b i} \overline{\mathbf{5}}_{j}^{H_{d}}+\frac{1}{8} \epsilon_{i j k l m} \mathbf{1 0}_{a}^{i j} \mathbf{1 0}_{b}^{k l} \mathbf{5}_{H_{u}}^{m}\right]
$$

where Latin indices $i, j, \cdots=1,2, \ldots 5$ are $\mathrm{SU}(5)$ indices. Since the terms in square brackets are not $a \leftrightarrow b$ symmetric, it is left understood that $\tilde{h}_{a b}$ (as well as $\tilde{f}_{a b}$ in the equation below) stands for the symmetrized coupling $\frac{1}{2}\left(\tilde{h}_{a b}+\tilde{h}_{b a}\right)$. In eq. (2.5) we have introduced labels for the scalar representations $\mathbf{5}_{H_{u}}$ and $\overline{\mathbf{5}}^{H_{d}}$, in order to recall that the terms in square brackets correspond (in this order) to the neutrino Dirac coupling to the multiplet containing the $\mathrm{SU}(2)$ doublet $H_{u}$, to the usual charged lepton and $d$-quark couplings to the scalar multiplet containing the down type Higgs $H_{d}$, and to the $u$-quark Yukawa coupling. The projection of eq. (2.3) onto $\mathrm{SU}(5)$ representations yields [36]:

$$
\begin{aligned}
& -\mathcal{L}_{\mathbf{1 2 6}} \rightarrow-\mathcal{L}_{f}=i \sqrt{\frac{2}{15}} \tilde{f}_{b a}\left[-\sqrt{2} \mathbf{1}_{a} \mathbf{1}_{b} \mathbf{1}^{S}-\sqrt{3} \mathbf{1}_{a} \overline{\mathbf{5}}_{b i} \mathbf{5}_{\Sigma_{u}}^{i}+\mathbf{1}_{a} \mathbf{1 0} \mathbf{0}_{b}^{i j} \overline{\mathbf{1 0}}_{i j}^{\Delta}\right. \\
& -\frac{1}{8 \sqrt{3}} \epsilon_{i j k l m} \mathbf{1 0}_{a}^{i j} \mathbf{1 0}_{b}^{k l} \mathbf{5}_{\Sigma_{u}}^{m}+\mathbf{1 0}_{a}^{i j} \overline{\mathbf{5}}_{b k} \overline{\mathbf{4 5}}_{i j}^{\Sigma_{d} k} \\
& \left.-\overline{\mathbf{5}}_{a i} \overline{\mathbf{5}}_{b j} \mathbf{1 5 ^ { i j }}-\frac{1}{12 \sqrt{2}} \epsilon_{i j k l m} \mathbf{1 0}_{a}^{l m} \mathbf{1 0} \mathbf{0}_{b}^{r s} \mathbf{5 0} \mathbf{0}_{r s}^{i j k}\right] \text {. }
\end{aligned}
$$

The first three terms in square brackets involve the $\mathrm{SU}(5)$ singlet $\mathbf{1}=N$, and correspond respectively to the Majorana coupling to the $\mathrm{SU}(5)$ singlet scalar $\mathbf{1}^{S}$ that provides the $N$ masses, to a Dirac coupling to a second $u$-type scalar doublet $\Sigma_{u}$, and to an interaction term (the $\overline{\mathbf{1 0}}^{\Delta}$ contains only charged scalars). The terms in the second line couple quarks and leptons to the additional SU(2) scalar doublets $\Sigma_{u, d}$. The two terms in the third line do not involve $N$ and do not give corrections to the charged fermion masses. However, 15 contains an $\mathrm{SU}(2)$ triplet, and a small vev for its neutral component is often used to implement in $\mathrm{SO}(10)$ the type II seesaw. We have assumed from the start that neutrino masses are dominated by the type I seesaw, and thus these last two terms are not relevant for us and will be omitted in the following equations. Then, the relevant $\mathrm{SU}(5) \rightarrow \mathrm{SU}(2)_{L} \times \mathrm{SU}(3)$ branching rules for projecting eqs. $(2.5)-(2.6)$ onto $\mathcal{G}_{\mathrm{SM}}$ multiplets are:

$$
\begin{aligned}
\overline{\mathbf{5}} & =(\mathbf{2}, \mathbf{1}) \oplus(\mathbf{1}, \overline{\mathbf{3}}), \\
\mathbf{1 0} & =(\mathbf{1}, \mathbf{1}) \oplus(\mathbf{1}, \overline{\mathbf{3}}) \oplus(\mathbf{2}, \mathbf{3}), \\
\overline{\mathbf{4 5}} & =(\mathbf{2}, \mathbf{1}) \oplus \ldots,
\end{aligned}
$$

where the $\overline{\mathbf{4 5}}$ contains additional coloured multiplets that play no role in our analysis. Written explicitly, the embedding of the SM fermions into the $\overline{\mathbf{5}} \oplus \mathbf{1 0}$ of $\mathrm{SU}(5)$ is:

$$
\overline{\mathbf{5}}=\left(\begin{array}{c}
d_{1}^{c} \\
d_{2}^{c} \\
d_{3}^{c} \\
e^{-} \\
-\nu
\end{array}\right), \quad \mathbf{1 0}=\frac{1}{\sqrt{2}}\left(\begin{array}{ccccc}
0 & u_{3}^{c} & -u_{2}^{c} & u_{1} & d_{1} \\
-u_{3}^{c} & 0 & u_{1}^{c} & u_{2} & d_{2} \\
u_{2}^{c} & -u_{1}^{c} & 0 & u_{3} & d_{3} \\
-u_{1} & -u_{2} & -u_{3} & 0 & e^{c} \\
-d_{1} & -d_{2} & -d_{3} & -e^{c} & 0
\end{array}\right)
$$


which also fixes the assignments for the scalars in the $\mathbf{5}, \overline{\mathbf{5}}$ and $\overline{\mathbf{1 0}}$ of $\mathrm{SU}(5)$. As regards the $\overline{\mathbf{4 5}}$, it is contained in the reducible three index representation antisymmetric in the upper indices $\mathbf{5 0}_{k}^{i j}=\left(\mathbf{5}^{i} \times \mathbf{5}^{j}\right)_{a} \times \overline{\mathbf{5}}_{k}$, and it can be singled out by subtracting the $\sum_{i} \mathbf{5 0}_{i}^{i j}$ trace part (that transforms as an irreducible $\mathbf{5}$ ), that is by imposing the five constraints $\sum_{i} \mathbf{4 5}_{i j}^{i}=0(j=1,2 \ldots, 5)$. The projection of eqs. $(2.5)-(2.6)$ onto $\mathcal{G}_{\mathrm{SM}}$ multiplets is now straightforward, and yields:

$$
\begin{aligned}
-\mathcal{L}_{Y}=2 i \sqrt{2} \tilde{h}_{a b}[ & \left.-N_{a} \ell_{b} H_{u}+e_{a}^{c} \ell_{b} H_{d}+Q_{a} d_{b}^{c} H_{d}+Q_{a} u_{b}^{c} H_{u}\right]-i \sqrt{\frac{2}{15}} \tilde{f}_{a b}\left[\sqrt{2} N_{a} N_{b} S\right. \\
& \left.+\sqrt{3} N_{a} \ell_{b} \Sigma_{u}+\frac{1}{\sqrt{3}} Q_{a} u_{b}^{c} \Sigma_{u}-e_{a}^{c} \ell_{b} \Sigma_{d}+\frac{1}{3} Q_{a} d_{b}^{c} \Sigma_{d}-2 N_{a} e_{b}^{c} \Delta\right]
\end{aligned}
$$

where we have omitted several couplings to heavy (GUT scale) coloured scalars. Let us draw the attention to the last term involving the scalar field $\Delta$ with charge -1 , since it is going to be relevant in what follows. By redefining:

$$
h=2 i \sqrt{2} \tilde{h}, \quad f=-\frac{i}{3} \sqrt{\frac{2}{15}} \tilde{f},
$$

we can finally rewrite eq. (2.9) as:

$$
\begin{aligned}
-\mathcal{L}_{Y}= & 3 \sqrt{2} f_{a b} N_{a} N_{b} S+e_{a}^{c}\left[h_{a b} H_{d}-3 f_{a b} \Sigma_{d}\right] \ell_{b}+Q_{a}\left[h_{a b} H_{d}+f_{a b} \Sigma_{d}\right] d_{b}^{c} \\
& +Q_{a}\left[h_{a b} H_{u}+\sqrt{3} f_{a b} \Sigma_{u}\right] u_{b}^{c}-N_{a}\left[h_{a b} H_{u}-3 \sqrt{3} f_{a b} \Sigma_{u}\right] \ell_{b}-6 f_{a b} N_{a} e_{b}^{c} \Delta .
\end{aligned}
$$

\section{Leptogenesis Lagrangian and CP asymmetries}

The relevant couplings to compute the leptogenesis CP asymmetries and washout scatterings can be read off eq. (2.11) after spelling out a few points characterizing the scenario.

- The heavy RH neutrinos $N$ acquire an intermediate scale mass via the vev of the SM singlet scalar $S$ that sits in the $\overline{\mathbf{1 2 6}}$. We define:

$$
\sigma=\sqrt{\left\langle S^{\dagger} S\right\rangle}
$$

Under $\mathcal{G}_{P S}$ the $\overline{\mathbf{1 2 6}}$ branches to $(\mathbf{1}, \mathbf{1}, \overline{\mathbf{6}}) \oplus(\mathbf{3}, \mathbf{1}, \mathbf{1 0}) \oplus(\mathbf{1}, \mathbf{3}, \overline{\mathbf{1 0}}) \oplus(\mathbf{2}, \mathbf{2}, \mathbf{1 5}) . S$ is the neutral component and $\mathrm{SU}(3)_{c}$ singlet of $(\mathbf{1}, \mathbf{3}, \overline{\mathbf{1 0}})$ so that $\sigma$ also breaks $\mathcal{G}_{P S} \rightarrow \mathcal{G}_{\mathrm{SM}}$.

- The scalar field $\Delta$ is the charge -1 component of the same multiplet, and is expected to acquire an intermediate scale mass as well. We thus need to allow for the possibility $M_{N}>M_{\Delta}$, i.e. that the decay channel $N \rightarrow e^{c} \Delta$ is open. The possibility of $N$ decays into $\mathrm{SU}(2)_{L}$ singlet leptons and scalars was already studied in [37] (although not in relation with $\mathrm{SO}(10)$ ) and it was found to be potentially interesting for leptogenesis. One of the reasons is that the specific decays into $e_{1}^{c}$, defined as the $\mathrm{SU}(2)$ singlet lepton most weakly coupled to the $N$ 's, would generate an asymmetry that remains completely decoupled from the thermal bath, and in particular unaffected, even indirectly, by potentially large $\ell_{1} H \leftrightarrow N$ washouts, since the Yukawa coupling relating $e_{1}^{c}$ and $\ell_{1}$ remains out of equilibrium down to temperatures $T \ll M_{N}$. 
- The scalar fields $\Sigma_{u, d}$ belong to $(\mathbf{2}, \mathbf{2}, \mathbf{1 5})$ of $\mathcal{G}_{P S}$ and have a mass unrelated to the vev $\sigma$. Naturalness considerations then suggest that $M_{\Sigma} \gg M_{N}$ in which case the decays $N \rightarrow \ell \Sigma_{u}$ are kinematically forbidden. Note however, that the neutral components of these bi-doublets will acquire induced vevs proportional to the EW vevs residing in the 10, via the coupling $(\overline{\mathbf{1 2 6}} \mathbf{1 2 6})$ (126 10) [8]. These induced vevs are of fundamental importance to achieve the correct fermion mass relations.

- In general, $\mathrm{SO}(10)$ fits to SM observables produce spectra with heavy Majorana neutrino masses in the range $10^{9}-10^{12} \mathrm{GeV}[1,12,14,19]$. The appropriate regime to study leptogenesis is then the three flavour regime [38-41], which thus requires considering the flavoured CP asymmetries and flavour-dependent washouts.

As we have already mentioned, at the unbroken $\mathrm{SO}(10)$ level one can always choose a basis in which one of the two matrices of Yukawa coupling is diagonal with real nonnegative entries, and we choose $h_{a b}=\hat{h}_{a} \delta_{a b}$, while $f$ remains a generic complex symmetric matrix. After intermediate $\mathrm{SO}(10) \rightarrow \mathcal{G}_{P S}$ breaking, the appropriate basis for leptogenesis is the basis of the $N$ 's mass eigenstates, defined via a rotation of the Majorana fields with a unitary matrix $W$ that brings the matrix $f$ in the first term in eq. (2.11) into diagonal form: $\hat{f}=W f W^{T}$. In this basis, the Lagrangian terms relevant for leptogenesis can be written as:

$$
-\mathcal{L}_{L G}=\frac{1}{2} M_{N_{j}} N_{j} N_{j}-N_{j}(W \hat{h})_{j \alpha} \ell_{\alpha} H_{u}-6 N_{j}\left(\hat{f} W^{*}\right)_{j \alpha} e_{\alpha}^{c} \Delta
$$

where

$$
M_{N}=6 \sqrt{2} \hat{f} \sigma .
$$

Since at the leptogenesis temperatures $T \sim M_{N} \ll M_{\Sigma}$ scatterings between leptons and $\Sigma_{d}$ do not occur, the flavour basis $\left\{\ell_{\alpha}, e_{\alpha}^{c}\right\}$ remains fixed by the leptons Yukawa interactions with the light $d$-type Higgs: $\hat{h}_{\alpha} e_{\alpha}^{c} \ell_{\alpha} H_{d}$. Here and below we denote with Latin subscripts $j, k, \ldots$ the heavy neutrinos mass eigenstates ordered from light $(j=1)$ to heavy $(j=3)$, and with Greek subscripts $\alpha, \beta, \ldots$ the lepton flavours, ordered according to the strength of their Yukawa couplings $\hat{h}_{1}<\hat{h}_{2}<\hat{h}_{3}$. It is worth noticing at this point, that due to the contribution of the $f$ couplings to the lepton masses after EW symmetry breaking, the SM mass eigenstates $e, \mu, \tau$ will not in general coincide with the leptogenesis flavour eigenstates $\ell_{1}, e_{1}^{c} ; \ell_{2}, e_{2}^{c} ; \ell_{3}, e_{3}^{c}$.

\subsection{CP violating asymmetries}

The CP violating asymmetries for $N_{j}$ decays into leptons of flavour $\alpha$ are defined in terms of decay widths in the usual way:

$$
\begin{aligned}
\epsilon_{j \alpha}^{H} & =\frac{1}{\Gamma_{\text {tot }}^{N_{j}}}\left(\Gamma_{\ell_{\alpha} H_{u}}^{N_{j}}-\Gamma_{\bar{\ell}_{\alpha} H_{u}^{*}}^{N_{j}}\right), \\
\epsilon_{j \alpha}^{\Delta} & =\frac{-1}{\Gamma_{\text {tot }}^{N_{j}}}\left(\Gamma_{e_{\alpha}^{c} \Delta}^{N_{j}}-\Gamma_{\bar{e}_{\alpha}^{c} \Delta^{*}}^{N_{j}}\right),
\end{aligned}
$$


where superscripts (subscripts) denote initial (final) decay states, and

$$
\begin{aligned}
\Gamma_{\text {tot }}^{N_{j}} & =\frac{M_{N_{j}}}{16 \pi} D_{j}, \\
D_{j} & \equiv 2 \sum_{\alpha}\left|W_{j \alpha}\right|^{2} \hat{h}_{\alpha}^{2}+36 \hat{f}_{j}^{2},
\end{aligned}
$$

where the factor of two takes into account gauge multiplicities of the $\mathrm{SU}(2)$ doublets, while $6^{2}=36$ originates from the prefactor in eq. (3.2) for the SU(2) singlets term. Note that the asymmetry in eq. (3.5) is defined with an overall minus sign, that compensates the fact that in the production of $e^{c}$ lepton number is decreased by one unit. In defining the total width $\Gamma_{\text {tot }}^{N_{j}}$ eq. (3.6) we have neglected $W_{R}$ mediated three body decays $N \rightarrow e^{c} \bar{u}^{c} d^{c}$. Given that all the $N$ 's are strongly coupled to at least one lepton flavour (one Yukawa coupling is in fact related to the top Yukawa coupling) this is justified as long as $M_{W_{R}} \gtrsim M_{N}$.

Computation of the $\mathrm{CP}$ asymmetries can be carried out in the usual way [42]. In particular, in spite of the presence of the new decay channel $N \rightarrow e^{c} \Delta$, there are no new loop contributions. This is because $f$-related couplings appear in the loops in the combination $\sum_{\alpha}\left(\hat{f} W^{*}\right)_{j \alpha}\left(\hat{f} W^{*}\right)_{\alpha k}^{\dagger} \propto \delta_{j k}$ which vanishes for $k \neq j$. For the same reason, the total CP asymmetries in $N_{j} \rightarrow e_{\alpha}^{c} \Delta$ decays summed over flavours also vanish $\sum_{\alpha} \epsilon_{j \alpha}^{\Delta}=0$. Thus, the contribution of this channel is of the "purely flavoured leptogenesis" (PFL) type [43]. For the CP asymmetries we obtain:

$$
\begin{aligned}
\epsilon_{j \alpha}^{H} & =\frac{4 \hat{h}_{\alpha}^{2}}{16 \pi D_{j}} \sum_{\beta, k \neq j} \hat{h}_{\beta}^{2} \operatorname{Im}\left[W_{j \alpha} W_{k \alpha}^{*}\left(W_{j \beta} W_{k \beta}^{*} g^{S V}\left(x_{k j}\right)+W_{k \beta} W_{j \beta}^{*} g^{S^{\prime}}\left(x_{k j}\right)\right)\right], \\
\epsilon_{j \alpha}^{\Delta} & =\frac{72 \hat{f}_{j}}{16 \pi D_{j}} \sum_{\beta, k \neq j} \hat{f}_{k} \hat{h}_{\beta}^{2} \operatorname{Im}\left[W_{j \alpha}^{*} W_{k \alpha}\left(W_{j \beta} W_{k \beta}^{*} g^{S}\left(x_{k j}\right)+W_{k \beta} W_{j \beta}^{*} g^{S^{\prime}}\left(x_{k j}\right)\right)\right],
\end{aligned}
$$

where $x_{k j}=\frac{M_{k}^{2}}{M_{j}^{2}}=\frac{\hat{f}_{k}^{2}}{\hat{f}_{j}^{2}}$. The function $g^{S V}=g^{S}+g^{V}$ is the sum of the self energy and vertex loop functions:

$$
\begin{aligned}
g^{S} & =\frac{\sqrt{x}}{1-x} \rightarrow-\frac{1}{\sqrt{x}}-\frac{1}{x^{3 / 2}}+\ldots \\
g^{V} & =\sqrt{x}\left[1-(1+x) \ln \frac{1+x}{x}\right] \rightarrow-\frac{1}{2 \sqrt{x}}+\frac{1}{6 x^{3 / 2}}+\ldots,
\end{aligned}
$$

where the limiting expressions hold for $x \rightarrow \infty$. Let us note that for the $\epsilon_{j \alpha}^{\Delta}$ asymmetries there is no vertex contribution $g^{V}$. The second term in eq. (3.8) (which is sometimes denoted as the 'lepton number conserving term') involves the self energy function

$$
g^{S^{\prime}}(x)=\frac{1}{1-x} \rightarrow-\frac{1}{x}-\frac{1}{x^{2}}+\ldots
$$

and it does not contribute to the total asymmetry $\epsilon_{j}^{H}=\sum_{\alpha} \epsilon_{j \alpha}^{H}$. In fact by summing over flavour one obtains $\left|\left(W \hat{h}^{2} W^{\dagger}\right)_{j k}\right|^{2}$ which is real. We can conclude that the $g^{S^{\prime}}$ contribution is also of the PFL type $[41,42]$. It is interesting to note that while in $\epsilon_{1 \alpha}^{H}$ this term is in any 
case subdominant, given that (in the hierarchical limit) $g^{S^{\prime}}\left(x_{k 1}\right) / g^{S}\left(x_{k 1}\right) \sim M_{1} / M_{k} \ll 1$, it represents instead the dominant contribution to the $N_{1}$ CP asymmetry $\epsilon_{1 \alpha}^{\Delta}$. This is because at the leading order in $M_{1} / M_{k}=\hat{f}_{1} / \hat{f}_{k}$, which comes from the $g^{S}$ expansion, $\epsilon_{1 \alpha}^{\Delta}$ vanishes, so that $g^{S}$ contributes only at order $\left(\hat{f}_{1} / \hat{f}_{k}\right)^{3}$. Thus the first order contribution from $g^{S^{\prime}} \sim\left(\hat{f}_{1} / \hat{f}_{k}\right)^{2}$ is the dominant one. The fact that $\epsilon_{1 \alpha}^{\Delta}$ vanishes at first order in the expansion can be seen by extending the sum over $k$ in the first term of eq. (3.9) to include also $k=1$ (the added term is real and does not contribute). At leading order we obtain

$$
\sum_{k} \hat{f}_{k} W_{k \alpha} W_{k \beta}^{*} g^{S}\left(x_{k 1}\right) \quad \rightarrow \quad-\hat{f}_{1} \sum_{k} W_{k \alpha} W_{k \beta}^{*}=-\hat{f}_{1} \delta_{\alpha \beta}
$$

Thus the first term in eq. (3.9) is, at leading order, real $\propto\left|W_{j \alpha}\right|^{2}$ and gives no contribution to $\epsilon_{1 \alpha}^{\Delta}$ (for $\epsilon_{2,3}^{\Delta}$ there is no analogous result because the expansions eqs. (3.10)-(3.12) do not hold). This is yet another example of the various cancellations that follow as a consequence of the $\mathrm{SO}(10)$ Yukawa structure.

\subsection{Boltzmann Equations}

We are now ready to write the network of Boltzmann Equations (BE) for $\mathrm{SO}(10)$ leptogenesis. In full generality, we need three $\mathrm{BE}$ for the evolution of the $N_{1,2,3}$ number densities and three for the usual anomaly free lepton charges $\Delta_{\alpha}=\Delta B / 3-\Delta L_{\alpha}[38,39]$. There is, however, another quantity that in the limit of vanishing $N$ couplings is conserved: it corresponds to the $\mathrm{U}(1)_{e_{1}}$ generator of phase transformations for the $\mathrm{SU}(2)$ singlet lepton field $e_{1}$ defined as the one with the smallest coupling to the Higgs. Since $e_{1}$ is an $\mathrm{SU}(2)$ singlet it does not participate in sphaleron processes. At the relevant temperatures $T \sim M_{N}$ (with $10^{9} \mathrm{GeV} \lesssim M_{N} \ll M_{\Sigma}$ ) its interactions with the Higgs field $H_{d}$ are completely out of equilibrium, and there are also no interactions with the massive scalars $\Sigma_{d}$ which have already disappeared from the thermal bath. Then in the effective theory governing this regime we can set $\hat{h}_{1}^{\left(e_{1}\right)}, \hat{f}_{1}^{\left(e_{1}\right)} \rightarrow 0$ [44]. This results in the $\mathrm{U}(1)_{e_{1}}$ invariance and in a fourth conserved charge $\Delta_{4}=\Delta e_{1}$. Let us thus define the following charge densities (normalized to the entropy density) that are violated just by $N$ 's interactions while are conserved by all the reactions which are in thermal equilibrium (including non-perturbative sphaleron processes):

$$
\begin{aligned}
Y_{\Delta_{1}} & =\frac{1}{3} Y_{\Delta B}-2 Y_{\Delta \ell_{1}} \\
Y_{\Delta_{2}} & =\frac{1}{3} Y_{\Delta B}-\left(2 Y_{\Delta \ell_{2}}+Y_{\Delta e_{2}}\right) \\
Y_{\Delta_{3}} & =\frac{1}{3} Y_{\Delta B}-\left(2 Y_{\Delta \ell_{3}}+Y_{\Delta e_{3}}\right), \\
Y_{\Delta_{4}} & =Y_{\Delta e_{1}}
\end{aligned}
$$

To write down the network of flavoured BE as a closed system, one needs to express the density asymmetries of the five leptons $Y_{\Delta l} \equiv\left\{Y_{\Delta \ell_{1}}, Y_{\Delta \ell_{2}}, Y_{\Delta \ell_{3}}, Y_{\Delta e_{2}}, Y_{\Delta e_{3}}\right\}$ and of the up-type Higgs $Y_{\Delta H_{u}}$, which weight the washout terms, as linear combinations of the four 
$\left(Y_{\Delta}\right)_{q}$, that is:

$$
\begin{aligned}
\left(Y_{\Delta l}\right)_{p} & =A_{p q},\left(Y_{\Delta}\right)_{q} \\
Y_{\Delta H_{u}} & =C_{q}\left(Y_{\Delta}\right)_{q} .
\end{aligned}
$$

Note in passing that while $H_{u}$ develops an asymmetry, there is no asymmetry for the scalar $\Delta$. This is because the $\Delta$ are produced in decays for which the total asymmetry vanishes, and in addition there are no other scatterings in the plasma involving the $\Delta$ and SM or other particles with intermediate scale masses. Therefore the $\Delta$ are not subject to any chemical potential equilibrium condition that could result in $Y_{\Delta_{\Delta}} \neq 0$.

The $5 \times 4 A$ matrix and the $C$-vector in eq. (3.18) can be obtained by solving the system of linear constraints corresponding to in-equilibrium spectator reactions (Yukawa related scatterings, EW and QCD sphalerons [45]) and exactly conserved quantities (hypercharge). This yields:

$$
A=\left(\begin{array}{cccc}
-\frac{93}{220} & \frac{12}{220} & \frac{12}{220} & -\frac{2}{220} \\
\frac{9}{240} & -\frac{76}{240} & \frac{4}{240} & \frac{6}{240} \\
\frac{9}{240} & \frac{4}{240} & -\frac{76}{240} & \frac{6}{240} \\
\frac{21}{264} & -\frac{68}{264} & \frac{20}{264} & -\frac{18}{264} \\
\frac{21}{264} & \frac{20}{264} & -\frac{68}{264} & -\frac{18}{264}
\end{array}\right), \quad C=\frac{1}{880}(-37,-52,-52,+82)
$$

The final $B-L$ asymmetry resulting from leptogenesis is given by the sum $Y_{\Delta_{B-L}}=$ $\sum_{q}\left(Y_{\Delta}^{\infty}\right)_{q}$ where $\left(Y_{\Delta}^{\infty}\right)_{q}$ are obtained by integrating the BE. Finally, with two Higgs doublets, the relation between the baryon asymmetry and $Y_{\Delta_{B-L}}$ is:

$$
Y_{\Delta B}=\frac{8}{23} Y_{\Delta_{B-L}}
$$

The BE for the evolution of the $N_{j}$ densities and of the $\left(Y_{\Delta}\right)_{q}$ charge asymmetries, considering only decays and inverse decays read:

$$
\begin{aligned}
& \dot{Y}_{N_{j}}=-\gamma_{j}\left(\frac{Y_{N_{j}}}{Y_{N_{j}}^{\mathrm{eq}}}-1\right), \\
& \dot{Y}_{\Delta_{1}}=-\sum_{j}\left\{\epsilon_{j 1}^{H} \gamma_{j}\left(\frac{Y_{N_{j}}}{Y_{N_{j}}^{e q}}-1\right)-\frac{1}{2}\left(\frac{Y_{\Delta \ell_{1}}}{Y_{f}^{\mathrm{eq}}}+\frac{Y_{\Delta H_{u}}}{Y_{b}^{\mathrm{eq}}}\right) \gamma_{\ell_{1} H}^{N_{j}}\right\}, \\
& \dot{Y}_{\Delta_{2}}=-\sum_{j}\left\{\left(\epsilon_{j 2}^{H}+\epsilon_{j 2}^{\Delta}\right) \gamma_{j}\left(\frac{Y_{N_{j}}}{Y_{N_{j}}^{\mathrm{eq}}}-1\right)-\frac{1}{2}\left[\left(\frac{Y_{\Delta \ell_{2}}}{Y_{f}^{\mathrm{eq}}}+\frac{Y_{\Delta H_{u}}}{Y_{b}^{\mathrm{eq}}}\right) \gamma_{\ell_{2} H}^{N_{j}}+\frac{Y_{\Delta e_{2}}}{Y_{f}^{\mathrm{eq}}} \gamma_{e_{2} \Delta}^{N_{j}}\right]\right\}, \\
& \dot{Y}_{\Delta_{3}}=\dot{Y}_{\Delta_{2}}(2 \leftrightarrow 3), \\
& \dot{Y}_{\Delta_{4}}=\sum_{j}\left\{\epsilon_{j 1}^{\Delta} \gamma_{j}\left(\frac{Y_{N_{j}}}{Y_{N_{j}}^{e q}}-1\right)-\frac{1}{2} \frac{Y_{\Delta e_{1}}}{Y_{f}^{\mathrm{eq}}} \gamma_{e_{1} \Delta}^{N_{j}}\right\}
\end{aligned}
$$

where the time derivative is defined as $\dot{Y} \equiv s H z \frac{d}{d z} Y$ with $z=M_{1} / T, H \simeq 1.66 \sqrt{g_{*}} T^{2} / M_{P}$ the Hubble parameter and $s=2 \pi^{2} g_{*} T^{3} / 45$ the entropy density, with $g_{*}=110.75$ the 
number of relativistic degrees of freedom including two Higgs doublets. $Y_{b}^{\mathrm{eq}}=2 Y_{f}^{\mathrm{eq}}=$ $15 /\left(4 \pi^{2} g_{*}\right)$ are the boson and fermion relativistic equilibrium number densities normalized to the entropy density, $Y_{N_{j}}^{\text {eq }}=n_{N_{j}}^{\text {eq }} / s=45 z_{j}^{2} \mathcal{K}_{2}\left(z_{j}\right) /\left(2 \pi^{4} g_{*}\right)$ is the equilibrium density for the non-relativistic $N$ 's with $z_{j}=\sqrt{x_{j 1}} z, \mathcal{K}_{n}$ is the modified Bessel function of type $n$, $\gamma_{j}=n_{N_{j}}^{\text {eq }} \Gamma_{\text {tot }}^{N_{j}} \mathcal{K}_{1}\left(z_{j}\right) / \mathcal{K}_{2}\left(z_{j}\right)$ is the thermal average of the total decay rate $\Gamma_{\text {tot }}^{N_{j}}$ eq. (3.6), and the $\gamma_{\ldots j}^{N_{j}}$ are similarly thermal averages of the $N_{j}$ partial decay rates.

\section{Relating leptogenesis parameters to observables}

To estimate the baryon asymmetry yield of the minimal $\mathrm{SO}(10)$ model we need the numerical values of the partial decay widths and CP asymmetries, and to compute these quantities we need to know the values of the Yukawa coupling matrices and of the vev $\sigma$ that fixes the scale of the $N$ 's masses. As we will now argue, the values of these parameters can be fixed almost univocally in terms of measured low energy observables, with only one single high energy parameter left free.

Let us define for the up- and down- type Higgs doublets (denoted with subscripts $q=u, d)$ the following vev-related quantities:

$$
\begin{aligned}
& v_{q}=\sqrt{\left\langle H_{q}^{\dagger} H_{q}\right\rangle+\left\langle\Sigma_{q}^{\dagger} \Sigma_{q}\right\rangle}, \\
& t_{\beta} \equiv \tan \beta=\frac{v_{u}}{v_{d}} \\
& c_{q} \equiv \cos \theta_{q}=\frac{\sqrt{\left\langle H_{q}^{\dagger} H_{q}\right\rangle}}{v_{q}} \\
& s_{q} \equiv \sin \theta_{q}=\frac{\sqrt{\left\langle\Sigma_{q}^{\dagger} \Sigma_{q}\right\rangle}}{v_{q}} \\
& t_{q} \equiv \tan \theta_{q}=\frac{s_{q}}{c_{q}}
\end{aligned}
$$

with

$$
\begin{aligned}
c_{q}^{2}+s_{q}^{2} & =1, \\
v_{u}^{2}+v_{d}^{2} & =v^{2}=\left(\sqrt{2} G_{F}\right)^{-1},
\end{aligned}
$$

and $G_{F}$ the Fermi constant. After EW symmetry breaking, the fermion mass parameters can be read off from eq. (2.11) by projecting the scalar bi-doublets fields $H_{u, d}$ and $\Sigma_{u, d}$ on their vevs. This yields:

$$
\begin{aligned}
\frac{1}{v} M_{\ell} & =c_{\beta}\left[\hat{h} c_{d}-3 f s_{d}\right] \equiv \hat{H}-3 F, \\
\frac{1}{v} M_{d} & =c_{\beta}\left[\hat{h} c_{d}+f s_{d}\right] \equiv \hat{H}+F, \\
\frac{1}{v} M_{u} & =s_{\beta}\left[\hat{h} c_{u}+\sqrt{3} f s_{u} e^{i \delta_{u}}\right] \equiv r(\hat{H}+s F), \\
-\frac{1}{v} M_{D} & =s_{\beta}\left[\hat{h} c_{u}-3 \sqrt{3} f s_{u} e^{i \delta_{u}}\right] \equiv r(\hat{H}-3 s F),
\end{aligned}
$$


with $M_{\ell, d, u}$ the charged leptons, $d$ - and $u$-type quarks mass matrices, and $M_{D}$ the Dirac mass matrix for the neutrinos. On the right-hand side (r.h.s. ) of the first equalities we have written the mass matrices keeping distinguished the vevs and the Yukawa couplings; this is needed to write down leptogenesis related quantities. The r.h.s. of the second equalities is instead written following a commonly adopted parameterization [1, 12, 14, 46-48], which is best suited for $\mathrm{SO}(10)$ fits to low energy data. In these notations the relation with the non-diagonal $\left(\mathcal{M}_{N}\right)$ and diagonal $\left(M_{N}\right)$ Majorana neutrino mass matrix can be written as:

$$
\mathcal{M}_{N}=W^{\dagger} M_{N} W^{*}=6 \sqrt{2} \sigma f=r_{R}^{-1} F .
$$

Thus, once $F$ is given, the unitary matrix $W$ can be determined from the requirement that it brings $F$ into its diagonal form with real non-negative entries. Here we follow in particular the conventions used in ref. [1] with $\hat{H}$ diagonal with 3 real non-negative entries, $F$ complex symmetric with $6+6$ (real + imaginary) parameters, $r$ and $r_{R}$ real quantities and $s \equiv|s| e^{i \delta_{s}}$ complex, for a total of 19 parameters (12 real and 7 imaginary). The low energy data set consists of 9 charged fermion masses, 2 neutrinos mass squared differences, $3+3$ quark and lepton mixing angles and 1 CKM phase, for a total of 18 parameters (17 real and 1 imaginary). Although the number of parameters exceeds by one the number of constraints from the data, $\mathrm{SO}(10)$ numerical fits are able to determine all 19 parameters (and thus to yield also predictions for yet unmeasured quantities like the Pontecorvo-MakiNakagawa-Sakata (PMNS) Dirac phase and the $0 \nu 2 \beta$ effective neutrino mass $[1,14]$ ). This is due in part to the nonlinearity of the relations that connect $\left\{\hat{H}, F, r, r_{R}, s\right\}$ to the data, and also to the fact that the number of real observables (moduli) is sizeably larger than that of real free parameters. All in all, fits to low energy data are able to fix the numerical values of the whole set $\left\{\hat{H}, F, r, r_{R}, s\right\}$. We want to relate these numerical quantities to our Yukawa parameters $\hat{h}, \hat{f}, W$ (two diagonal real matrices and one unitary, for a total of $3+3+(3+6)=9$ real +6 imaginary parameters) and to the $4+1$ vevs-related quantities $\sigma, t_{\beta}, s_{d}, s_{u} e^{i \delta_{u}}$, defined in the following way: the vevs $\left\langle H_{u, d}\right\rangle \propto c_{u, d}$ have one common phase [8] that can be reabsorbed redefining the remaining ones, and the phase of $s_{d}$ can be absorbed into $W^{*}$ by redefining $\delta_{u}$ and $\sigma$. As regards $\sigma$, it is in general complex, but its overall phase does not affect low energy data or leptogenesis, and therefore for simplicity we take $\sigma$ to be real. With respect to the parameterization in the r.h.s. of eqs. (4.8)-(4.12) we have one additional real parameter, that can be identified via the relation $F \leftrightarrow f \cdot s_{d}$. While low energy data are only sensitive to the product of the coupling matrices times the vev $\left\langle\Sigma_{d}\right\rangle$, leptogenesis is directly sensitive to the values of $f$ alone, and then it can allow to disentangle the Yukawa couplings from the vevs. More precisely, it is the value of $t_{d}$ (defined in eq. (4.5)) that can tell us which part of the corrections to quark/lepton universality is due to the $f$ Yukawas (whose size is relevant for leptogenesis) and which part is due to the vevs of the $\overline{\mathbf{1 2 6}}$ bi-doublets. We will then take $t_{d}$ as the new independent variable. All the other vev related quantities can then be written as:

$$
\begin{aligned}
& t_{u}=\frac{1}{\sqrt{3}}|s| t_{d}, \quad \delta_{u}=\delta_{s}, \\
& t_{\beta}=r \frac{c_{d}}{c_{u}}=r \sqrt{\frac{1+\frac{1}{3}|s|^{2} t_{d}^{2}}{1+t_{d}^{2}}},
\end{aligned}
$$




$$
\sigma=\frac{r_{R}^{-1}}{6 \sqrt{2}} \sqrt{\frac{t_{d}^{2}}{\left(1+r^{2}\right)+\left(1+\frac{1}{3} r^{2}|s|^{2}\right) t_{d}^{2}}} .
$$

Finally, for the two matrices $\hat{h}$ and $f$ we obtain:

$$
\begin{aligned}
& \hat{h}=\frac{1}{c_{\beta} c_{d}} \hat{H}=\hat{H} \sqrt{\left(1+r^{2}\right)+\left(1+\frac{1}{3} r^{2}|s|^{2}\right) t_{d}^{2}} \approx r \hat{H}, \\
& f=F \frac{1}{c_{\beta} s_{d}}=F \sqrt{\frac{\left(1+r^{2}\right)+\left(1+\frac{1}{3} r^{2}|s|^{2}\right) t_{d}^{2}}{t_{d}^{2}}} \approx \frac{r}{t_{d}} F,
\end{aligned}
$$

where the approximations hold for $r^{2} \gg 1,|s|^{2} \ll 1$ (which result from numerical fits) and $t_{d} \lesssim 1$ (which is favoured theoretically since $t_{d}$ is the ratio between an induced vev $\left\langle\Sigma_{d}\right\rangle$ and the EW vev $\left.\left\langle H_{d}\right\rangle\right)$. From eq. (4.14) we can see that in this approximation $t_{\beta} \approx r$, from eq. (4.16) we learn that the matrix $\hat{h}$ is not very sensitive with respect to changes in $t_{d}$ while, in contrast, from eq. (4.17) we see that $f$ is inversely proportional to $t_{d}$. This means that at fixed values of $F$, small vevs $\left\langle\Sigma_{d}\right\rangle$ imply large $f$-Yukawa couplings and this would render, among other things, more important the 'exotic' decay channel $N \rightarrow e^{c} \Delta$.

\section{Results}

From the equations above it should be clear that once the values of $\left\{\hat{H}, F, r, r_{R}, s\right\}$ are determined by fits to the low energy observables, the CP asymmetries eqs. (3.8)-(3.9) and the partial decay rates entering the BE eqs. (3.22)-(3.26) remain determined solely in terms of $t_{d}$. Note, however, that the heavy Majorana masses $M_{N}$ do not depend on this parameter (given that they do not depend on the doublets vevs) and thus the RH neutrino spectrum is univocally fixed solely by the low energy data. As we have already mentioned, the RH neutrino masses obtained from the numerical fits generally fall in the range $10^{9}-10^{12} \mathrm{GeV}$, which is a favourable one for leptogenesis, but it should be remarked that this type of results always implies a certain degree of tuning. The mass of the lightest Majorana neutrino $N_{1}$ would in fact more naturally lie in a mass range well below $10^{9} \mathrm{GeV}$. To show this, let us start by writing the seesaw formula in a generic basis:

$$
m_{\nu}=-M_{D} \mathcal{M}_{N}^{-1} M_{D},
$$

where $m_{\nu}$ and $M_{D}$ are respectively the light neutrino and the Dirac mass matrix (both symmetric). Let us further define the diagonal mass matrices $m_{\nu}^{d}$ and $M_{D}^{d}$ via (unitary) Takagi factorizations $M_{D}=V M_{D}^{d} V^{T}$ and $m_{\nu}=U m_{\nu}^{d} U^{T}$, where $U$ is the PMNS matrix. From the seesaw formula we have:

$$
\mathcal{M}_{N}^{-1}=-M_{D}^{-1} m_{\nu} M_{D}^{-1}=V^{*} \frac{1}{M_{D}^{d}} \mathcal{W} m_{\nu}^{d} \mathcal{W}^{T} \frac{1}{M_{D}^{d}} V^{\dagger}
$$

where we have defined $\mathcal{W}=V^{\dagger} U$. Let us now write:

$$
\mathcal{M}_{N}^{-1}\left(\mathcal{M}_{N}^{-1}\right)^{\dagger}=V^{*} \frac{1}{M_{D}^{d}} \mathcal{W} m_{\nu}^{d} \mathcal{W}^{T} \frac{1}{\left(M_{D}^{d}\right)^{2}} \mathcal{W}^{*} m_{\nu}^{d} \mathcal{W}^{\dagger} \frac{1}{M_{D}^{d}} V^{T}
$$


Taking the trace of this equation yields:

$$
\frac{\kappa}{M_{1}^{2}}=\sum_{i j} \frac{1}{\left(M_{D_{i}}^{d} M_{D_{j}}^{d}\right)^{2}}\left|\left(\mathcal{W} m^{d} \mathcal{W}^{T}\right)_{i j}\right|^{2}
$$

where in the left-hand side the sum of the squares of the inverse $\mathrm{RH}$ neutrino masses has been expressed as $\sum_{i} \frac{1}{M_{i}^{2}}=\frac{\kappa}{M_{1}^{2}}$ with $M_{1}$ the mass of the lightest RH neutrino and $\kappa \equiv 1+\frac{M_{1}^{2}}{M_{2}^{2}}+\frac{M_{1}^{2}}{M_{3}^{2}}$ ranging between 1 (strong hierarchy: $M_{1} \ll M_{2,3}$ ) and 3 (degeneracy: $M_{1} \sim M_{2} \sim M_{3}$ ). The RH side of eq. (5.4) is a sum of positive definite terms, and is thus larger than each single term. Let us take as dominant the one involving $\left(M_{D_{1}}^{d}\right)^{4}$ at the denominator. We obtain: ${ }^{2}$

$$
M_{1}<\sqrt{\kappa} \frac{\left(M_{D_{1}}^{d}\right)^{2}}{\left|\sum_{i} \mathcal{W}_{1 i}^{2} m_{i}^{d}\right|}
$$

Approximate quark-lepton Yukawa universality suggests $M_{D_{1}}^{d} \sim m_{u p}$. On the other hand $\mathcal{W}$ is the product of the PMNS matrix $U$ and of the matrix $V$ that, in the basis in which the down-type Yukawa couplings are diagonal, should be approximately given by the CKM matrix. This implies that $\mathcal{W}$ should depart sizeably from a pure diagonal form, and thus the denominator in eq. (5.5) is bounded from below. For example, for $\left|\sum_{i} \mathcal{W}_{1 i}^{2} m_{i}^{d}\right| \approx 10^{-3} \mathrm{eV}$, $\kappa \sim 3$ and $M_{D_{1}}^{d} \sim m_{u p} \sim 4 \mathrm{MeV}$, the upper bound $M_{1} \lesssim 3 \times 10^{7} \mathrm{GeV}$ is obtained. Departures from quark-lepton Yukawa universality can at most yield a factor of a few enhancement of $M_{D_{1}}^{d}$ with respect to $m_{u p}$, and therefore eq. (5.5) can give an estimate of the amount of tuning required to enforce cancellations in $\left|\sum_{i} \mathcal{W}_{1 i}^{2} m_{i}^{d}\right|$ such that $M_{1}$ can be lifted into the $10^{9}-10^{12} \mathrm{GeV}$ range. If from one hand it is somewhat unpleasant that the numerical values that we will use result from a certain amount of tuning in fitting $\mathrm{SO}(10)$ parameters, on the other hand we find intriguing that, without any knowledge of what is required for leptogenesis to be successful, low energy data alone force all the $M_{j}$ 's in the correct ballpark.

By means of numerical integration of the BE (3.22)-(3.26) it is now possible to verify if a set of fitted data points $\left\{\hat{H}, F, r, r_{R}, s\right\}$ can yield an amount of baryon asymmetry in agreement with observations. We use the most recent combined Planck and WMAP CMB measurements $[50,51]$, which yield at $95 \%$ c.l.

$$
Y_{\Delta B}^{\mathrm{CMB}}=(8.58 \pm 0.22) \times 10^{-11} .
$$

We take the $\left\{\hat{H}, F, r, r_{R}, s\right\}$ data points from the fits of Dueck and Rodejohann (DR) [1] to non-supersymmetric $\mathrm{SO}(10)$ with scalars in $\mathbf{1 0}+\overline{\mathbf{1 2 6}}$, that are labeled as MN (minimal non-supersymmetric).

Starting from the low energy data set (SM fermion masses and mixings) at the scale $\mu=M_{Z}$, DR perform two different types of fits to the MN model. In the first approach, which they denote as MN-RGE, the observable are evolved from the high energy scale down to $M_{Z}$, integrating out the heavy neutrinos $N_{j}$ one by one at the appropriate scale. The outcome of the running is then compared with the experimental data. This is the most

\footnotetext{
${ }^{2} \mathrm{~A}$ similar result is derived in the appendix of ref. [49].
} 
sophisticated approach, and in particular is expected to yield a more reliable fit to the heavy neutrino masses. This, besides having sizeable effects on the neutrino parameters [52], is a quite crucial ingredient in leptogenesis. The $N$ spectrum obtained with this procedure is:

$$
\left\{M_{N_{1}}, M_{N_{2}}, M_{N_{3}}\right\}=\left\{1.2 \times 10^{11}, 2.0 \times 10^{11}, 3.6 \times 10^{12}\right\} \mathrm{GeV} .
$$

As regards the numerical values of the set $\left\{\hat{H}, F, r, r_{R}, s\right\}$, they can be found in appendix A of [1] labeled as MN-RGE and are not recopied here. The main approximation in the DR analysis is that of neglecting effects of the intermediate scale states on gauge coupling unification and on the running of the Yukawa matrices, and it is quite hard to estimate the related uncertainty on the fitted parameters. ${ }^{3}$

Since, as said above, the details of the $N$ 's mass spectrum is one of the most influential ingredient for the outcome of leptogenesis, we will present our results allowing for a $3 \%$ fluctuation around the central values in eq. (5.7). While we make no claim that this fluctuation is accounting for the aforementioned theoretical uncertainty, it can still be illustrative of the sensitivity of the results on changes in the details of the $N$ 's spectrum.

The second approach followed by DR, that they denote as MN-noRGE, is based on a direct fit to the low energy neutrino parameters, and to the GUT scale values of the charged fermion observables, evolved to the high scale ignoring the effects of non-degenerate $\mathrm{RH}$ neutrinos. As it is clearly explained in the DR paper, this second approach cannot be considered fully consistent, however it provides a second reference point for our study which allows for an important comparison for the outcome of leptogenesis. The $N$ 's mass spectrum for the MN-noRGE case is:

$$
\left\{M_{N_{1}}, M_{N_{2}}, M_{N_{3}}\right\}=\left\{1.5 \times 10^{10}, 7.2 \times 10^{11}, 5.5 \times 10^{12}\right\} \mathrm{GeV},
$$

while the full set $\left\{\hat{H}, F, r, r_{R}, s\right\}$ is again given in appendix $\mathrm{A}$ of [1].

Our results for the MN-RGE case are depicted in figure 1. We compare the value of $Y_{\Delta_{B-L}}$ obtained by integrating the BE eqs. (3.22)-(3.26), and plotted as a function of the vev ratio $t_{d}=\left[\frac{\left\langle\Sigma_{d}^{\dagger} \Sigma_{d}\right\rangle}{\left\langle H_{d}^{\dagger} H_{d}\right\rangle}\right]^{1 / 2}$ with the experimental value which is derived from eq. (5.6) via eq. (3.21), and that is represented by the horizontal grey band. The thick purple line depicts the results for the combined contributions of the $N_{j} \rightarrow \ell_{\alpha} H$ and $N_{j} \rightarrow e_{\alpha}^{c} \Delta$ channels and the purple band corresponds to a $3 \%$ variation in the values of the $N_{j}$ masses in eq. (5.7). We see that $Y_{\Delta_{B-L}}$ keeps growing with increasing values of $t_{d}$, that is with decreasing values of the $f$-couplings. The reason is that there is an overall contribution of the wrong sign from the $N_{j} \rightarrow e_{\alpha}^{c} \Delta$ channels which is sizeable for small $t_{d}$, while with decreasing values of the $f$ couplings it becomes less important. All in all, we see that in the theoretically favoured region $t_{d}<1$ ( i.e. $\left\langle\Sigma_{d}^{\dagger} \Sigma_{d}\right\rangle<\left\langle H_{d}^{\dagger} H_{d}\right\rangle$ ) the purple band fails to intersect the experimentally allowed grey region. Only in the shaded region, corresponding to values $t_{d}>1$, which are however theoretically questionable, the upper border of the purple band touches the grey band. The results for the contributions of the $N_{j} \rightarrow \ell_{\alpha} H$

\footnotetext{
${ }^{3}$ A preliminary tentative in this direction, although in a slightly different setup, has been done in [53], where it has been shown that threshold effects at the intermediate scale can produce effects on the fermion observables at the electroweak scale as large as $30 \%$.
} 


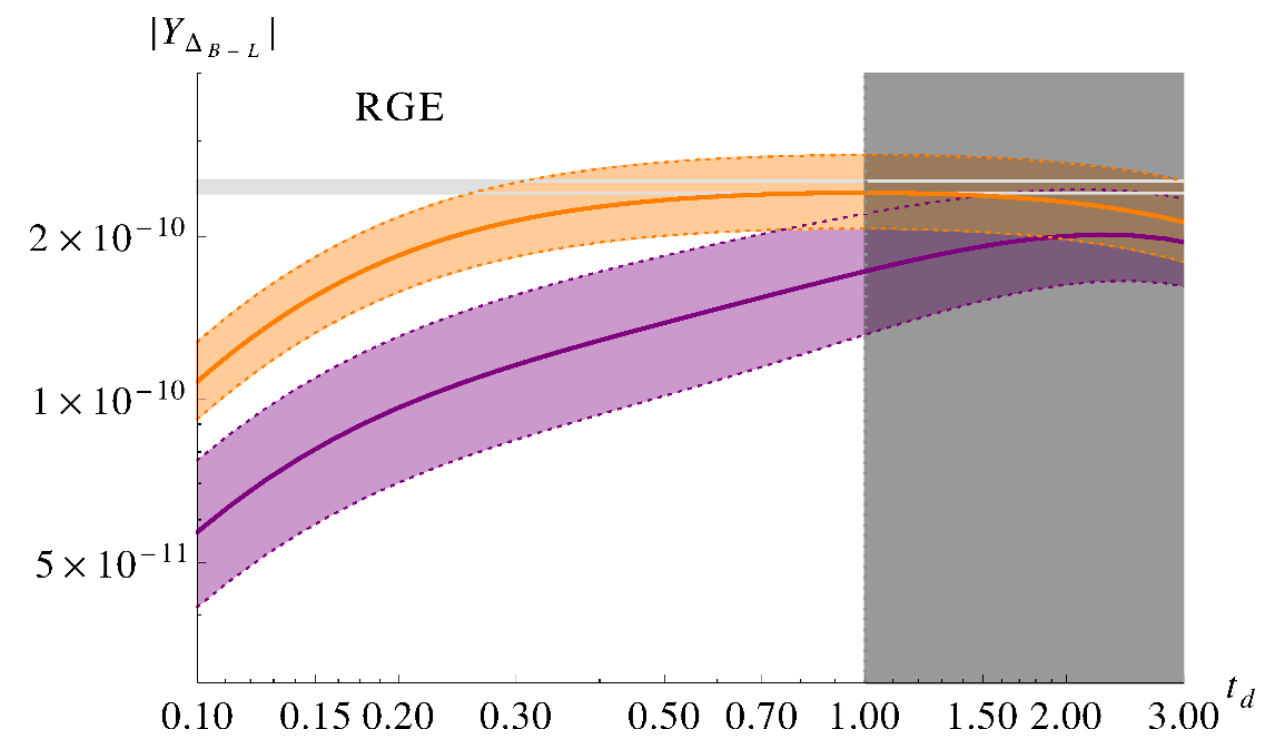

Figure 1. The $B-L$ asymmetry produced with the MN-RGE data set of ref. [1] plotted as a function of $t_{d}$. The horizontal grey band represents the experimental limit, the orange curve depicts the asymmetry generated via $N_{j} \rightarrow H \ell_{\alpha}$ decays alone, while the purple band includes also the $N_{j} \rightarrow \Delta e_{\alpha}^{c}$ decays. The width of the orange and purple bands correspond to a $3 \%$ variations in the $N_{j}$ masses.

channel alone are represented by the thick orange line. This corresponds to the situation in which the $\Delta$ scalar is heavier than $N_{1,2}\left(N_{3}\right.$ contributions to leptogenesis remain quite marginal), that is $M_{\Delta} \gtrsim 2.0 \times 10^{11} \mathrm{GeV}$, so that $N_{1,2}$ decays into $e_{\alpha}^{c} \Delta$ are kinematically forbidden. In this case the predicted central value touches the experimental band well within the region $t_{d}<1$, while the orange band nicely overlaps with the experimental band in the full interval $0.3 \lesssim t_{d} \lesssim 1$. It is also worth noticing that the maximum value of the $Y_{\Delta_{B-L}}$ asymmetry obtained in this model coincides rather precisely with the value obtained from observations. This is a bit intriguing, given that a priori this value could have been anything.

In summary, we find that the DR RGE fit to the minimal non-supersymmetric $\mathrm{SO}(10)$ model is fully consistent with the requirement that the observed value of the BAU is produced via leptogenesis, if the two conditions (i) $M_{\Delta} \gtrsim M_{N_{2}} \simeq 2.0 \times 10^{11} \mathrm{GeV}$ and (ii) $0.3 \lesssim\left[\frac{\left\langle\Sigma_{d}^{\dagger} \Sigma_{d}\right\rangle}{\left\langle H_{d}^{\dagger} H_{d}\right\rangle}\right]^{1 / 2} \lesssim 1$ are satisfied.

We present for comparison in figure 2 the results for the MN-noRGE data set. In this case the spectrum is sufficiently hierarchical (see eq. (5.8)) that leptogenesis is largely dominated by $N_{1}$ dynamics. The contribution of the $N_{j} \rightarrow \ell_{\alpha} H$ channels alone, represented by the thick orange line, remains well below the $Y_{\Delta_{B-L}}$ experimental band even in the $t_{d}>1$ region. In this case the effect of the $3 \%$ variations in the $N$ 's mass values is much milder than in the previous case, and this can be traced to the larger mass hierarchy and to $N_{1}$ dominance. Adding the contributions of the $N_{j} \rightarrow e_{\alpha}^{c} \Delta$ channels (the purple band and thick central line) has the striking effect of yielding a sizeably larger asymmetry, which is 


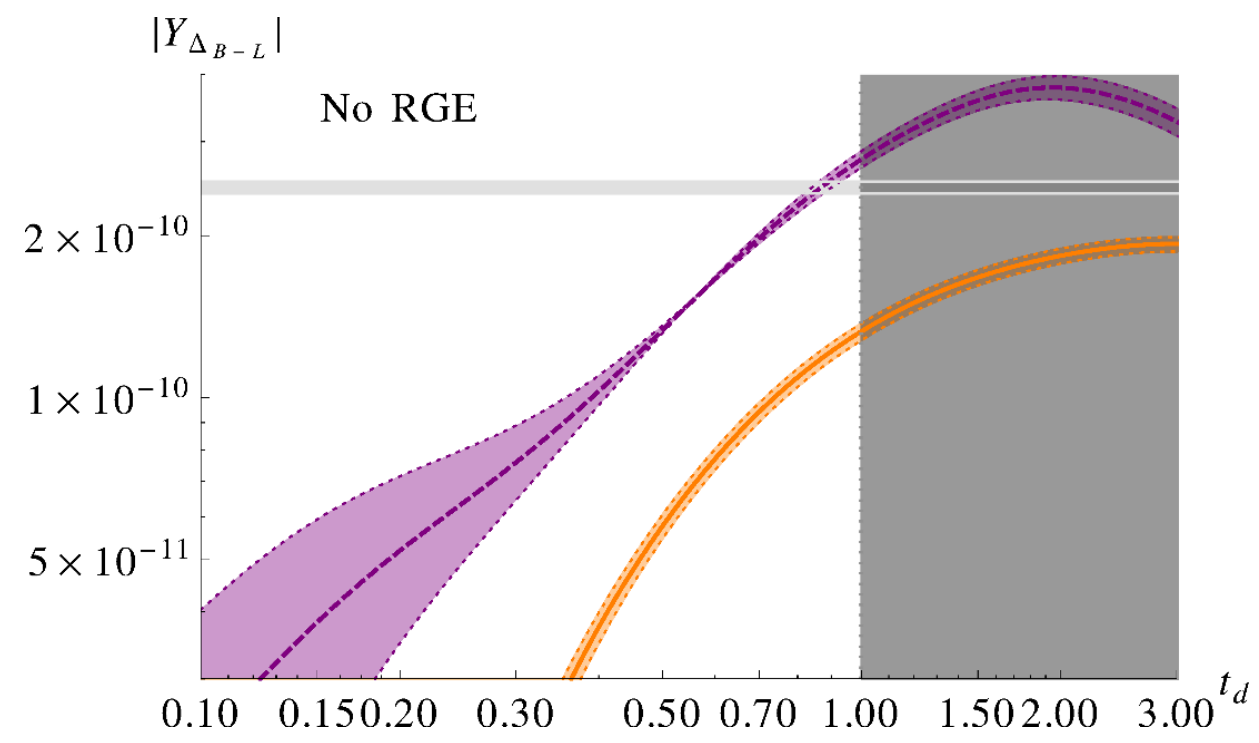

Figure 2. Same than figure 1 for the MN-noRGE data set of ref. [1]. The thick dashed line within the purple band indicates a wrong sign asymmetry.

however of the wrong sign (this is depicted in the figure by means of a dashed, rather than continuous, line). The fact that the $N_{j} \rightarrow e_{\alpha}^{c} \Delta$ contributions become particularly large with increasing $t_{d}$ is due to the fact that in this regime all the $N_{1} \rightarrow e_{\alpha}^{c} \Delta$ decay channels enter the weak washout regime, and washout effects keep decreasing as $t_{d}$ increases. $N_{1} \rightarrow \ell_{1,2} H$ decays also remain in the weak washout regime as long as $t_{d} \lesssim 3$, while $\ell_{3}$ is sufficiently strongly coupled to $N_{1}$ to ensure a thermal abundance for the RH neutrino independently of initial conditions. We also see how the purple bands widens at small values of $t_{d}$ (i.e. large values of the $f$-couplings). This is due to the vanishing of the first order $\mathcal{O}\left(M_{1} / M_{k}\right)$ contribution to $\epsilon_{1 \alpha}^{\Delta}$ that we have discussed at the end of section 3.1, which results in an enhanced sensitivity to the $M_{1} / M_{k}$ ratio. Let us also remark at this point that the sign of $Y_{\Delta_{B-L}}$ can be reverted (and the correct sign and size can thus be obtained) by simply reverting the signs of all the imaginary parts of the $F$ and $s$ parameters. While this will leave untouched the predictions for the fermion masses and mixings, it will result in the wrong sign for the CKM matrix $\delta_{C K M}$. Thus, it is just the interplay between $Y_{\Delta_{B-L}}$ and $\delta_{C K M}$ which allows to rule out the MN-noRGE data set with the contributions of the $N_{j} \rightarrow e_{\alpha}^{c} \Delta$ decays included.

In summary, while the results of the DR analysis [1] indicate that the MN-noRGE data set gives a better fit to the low energy data than the (more reliable) MN-RGE data set, we can conclude that MN-noRGE fails to produce a sufficiently large baryon asymmetry, and thus it does not pass the leptogenesis test.

\section{Concluding remarks}

In this work we have considered leptogenesis in a non-supersymmetric SO(10) GUT with fermion masses from the $\mathbf{1 0} \oplus \overline{\mathbf{1 2 6}}$ Higgs representations, which can (i) fit well all the 
low energy data, (ii) successfully account for unification of the gauge couplings, and (iii) allow for a sufficiently long lifetime of the proton. We have shown that, once the model parameters are fixed in terms of measured low energy observables, the requirement of successful leptogenesis can fix the only one remaining high energy parameter. We have highlighted that a new decay channel for the heavy Majorana neutrinos into the SU(2) singlet leptons $e^{c}$ is possible, and we have found that these decays can sizeably affect the size of the resulting baryon asymmetry. We have shown that the values of the model parameters obtained from the fits to low energy observables given in ref. [1] yield a baryon asymmetry in agreement with observations.

\section{Acknowledgments}

C.S.F. is supported by Fundação de Amparo à Pesquisa do Estado de São Paulo (FAPESP). C.S.F. acknowledges the hospitality of Universidad de Antioquia while this work was being completed. A.M. acknowledges fruitful correspondences with R. M. Syed, M. Malinsky and L. di Luzio. D.M. acknowledges MIUR for financial support under the program Futuro in Ricerca 2010 (RBFR10O36O). The work of E.N. is supported in part by the research grant "Theoretical Astroparticle Physics" number 2012CPPYP7 under the program PRIN 2012 funded by the Italian "Ministero dell'Istruzione, Università e della Ricerca" (MIUR) and by the INFN "Iniziativa Specifica" Theoretical Astroparticle Physics (TAsP-LNF).

Open Access. This article is distributed under the terms of the Creative Commons Attribution License (CC-BY 4.0), which permits any use, distribution and reproduction in any medium, provided the original author(s) and source are credited.

\section{References}

[1] A. Dueck and W. Rodejohann, Fits to SO(10) grand unified models, JHEP 09 (2013) 024 [arXiv: 1306.4468] [INSPIRE].

[2] C.S. Aulakh and R.N. Mohapatra, Implications of supersymmetric $\mathrm{SO}(10)$ grand unification, Phys. Rev. D 28 (1983) 217.

[3] T.E. Clark, T.-K. Kuo and N. Nakagawa, A SO(10) supersymmetric grand unified theory, Phys. Lett. B 115 (1982) 26 [INSPIRE].

[4] K. Matsuda, Y. Koide and T. Fukuyama, Can the $\mathrm{SO}(10)$ model with two Higgs doublets reproduce the observed fermion masses?, Phys. Rev. D 64 (2001) 053015 [hep-ph/0010026] [INSPIRE].

[5] K. Matsuda, Y. Koide, T. Fukuyama and H. Nishiura, How far can the SO(10) two Higgs model describe the observed neutrino masses and mixings?, Phys. Rev. D 65 (2002) 033008 [Erratum ibid. D 65 (2002) 079904] [hep-ph/0108202] [INSPIRE].

[6] T. Fukuyama and N. Okada, Neutrino oscillation data versus minimal supersymmetric SO(10) model, JHEP 11 (2002) 011 [hep-ph/0205066] [INSPIRE].

[7] F. del Aguila and L.E. Ibáñez, Higgs bosons in $\mathrm{SO}(10)$ and partial unification, Nucl. Phys. $\mathbf{B}$ 177 (1981) 60 [INSPIRE]. 
[8] K.S. Babu and R.N. Mohapatra, Predictive neutrino spectrum in minimal $\mathrm{SO}(10)$ grand unification, Phys. Rev. Lett. 70 (1993) 2845 [hep-ph/9209215] [INSPIRE].

[9] N.G. Deshpande, E. Keith and P.B. Pal, Implications of LEP results for $\mathrm{SO}(10)$ grand unification, Phys. Rev. D 46 (1993) 2261 [INSPIRE].

[10] L. Lavoura, Predicting the neutrino spectrum in minimal $\mathrm{SO}(10)$ grand unification, Phys. Rev. D 48 (1993) 5440 [hep-ph/9306297] [INSPIRE].

[11] S. Bertolini, L. Di Luzio and M. Malinsky, Intermediate mass scales in the non-supersymmetric $\mathrm{SO}(10)$ grand unification: a reappraisal, Phys. Rev. D 80 (2009) 015013 [arXiv: 0903.4049] [INSPIRE].

[12] G. Altarelli and D. Meloni, A non supersymmetric $\mathrm{SO}(10)$ grand unified model for all the physics below $M_{\mathrm{GUT}}$, JHEP 08 (2013) 021 [arXiv: 1305.1001] [INSPIRE].

[13] B. Bajc, A. Melfo, G. Senjanović and F. Vissani, Yukawa sector in non-supersymmetric renormalizable SO(10), Phys. Rev. D 73 (2006) 055001 [hep-ph/0510139] [InSPIRE].

[14] A.S. Joshipura and K.M. Patel, Fermion masses in SO(10) models, Phys. Rev. D 83 (2011) 095002 [arXiv: 1102 .5148] [INSPIRE].

[15] S. Bertolini, L. Di Luzio and M. Malinsky, Seesaw scale in the minimal renormalizable SO(10) grand unification, Phys. Rev. D 85 (2012) 095014 [arXiv:1202.0807] [INSPIRE].

[16] M. Fukugita and T. Yanagida, Baryogenesis without grand unification, Phys. Lett. B 174 (1986) 45 [inSPIRE].

[17] S. Davidson, E. Nardi and Y. Nir, Leptogenesis, Phys. Rept. 466 (2008) 105 [arXiv: 0802.2962] [INSPIRE].

[18] C.S. Fong, E. Nardi and A. Riotto, Leptogenesis in the universe, Adv. High Energy Phys. 2012 (2012) 158303 [arXiv: 1301.3062] [INSPIRE].

[19] F. Buccella, D. Falcone, C.S. Fong, E. Nardi and G. Ricciardi, Squeezing out predictions with leptogenesis from $\mathrm{SO}(10)$, Phys. Rev. D 86 (2012) 035012 [arXiv: 1203.0829] [INSPIRE].

[20] E. Nezri and J. Orloff, Neutrino oscillations versus leptogenesis in $\mathrm{SO}(10)$ models, JHEP 04 (2003) 020 [hep-ph/0004227] [INSPIRE].

[21] F. Buccella, D. Falcone and F. Tramontano, Baryogenesis via leptogenesis in $\mathrm{SO}(10)$ models, Phys. Lett. B 524 (2002) 241 [hep-ph/0108172] [INSPIRE].

[22] C.H. Albright and S.M. Barr, Resonant leptogenesis in a predictive $\mathrm{SO}(10)$ grand unified model, Phys. Rev. D 70 (2004) 033013 [hep-ph/0404095] [INSPIRE].

[23] O. Vives, Flavor dependence of CP asymmetries and thermal leptogenesis with strong right-handed neutrino mass hierarchy, Phys. Rev. D 73 (2006) 073006 [hep-ph/0512160] [INSPIRE].

[24] X.-d. Ji, Y.-c. Li, R.N. Mohapatra, S. Nasri and Y. Zhang, Leptogenesis in realistic $\mathrm{SO}(10)$ models, Phys. Lett. B 651 (2007) 195 [hep-ph/0605088] [INSPIRE].

[25] S.K. Majee, M.K. Parida, A. Raychaudhuri and U. Sarkar, Low intermediate scales for leptogenesis in SUSY SO(10) GUTs, Phys. Rev. D 75 (2007) 075003 [hep-ph/0701109] [INSPIRE].

[26] J.C. Romao, M.A. Tortola, M. Hirsch and J.W.F. Valle, Fermion masses, leptogenesis and supersymmetric SO(10) unification, Phys. Rev. D 77 (2008) 055002 [arXiv:0707.2942] [INSPIRE]. 
[27] X. Ji, Thermal leptogenesis in $\mathrm{SO}(10)$ GUT, Mod. Phys. Lett. A 23 (2008) 1464 [INSPIRE].

[28] T. Kikuchi, Leptogenesis in a perturbative $\mathrm{SO}(10)$ model, JHEP 09 (2008) 045 [arXiv:0802.3470] [INSPIRE].

[29] S.K. Majee, M.K. Parida and A. Raychaudhuri, Neutrino mass and low-scale leptogenesis in a testable SUSY SO(10) model, Phys. Lett. B 668 (2008) 299 [arXiv:0807.3959] [InSPIRE].

[30] A. Abada, P. Hosteins, F.-X. Josse-Michaux and S. Lavignac, Successful leptogenesis in $\mathrm{SO}(10)$ unification with a left-right symmetric seesaw mechanism, Nucl. Phys. B 809 (2009) 183 [arXiv:0808.2058] [INSPIRE].

[31] A. Romanino, Leptogenesis with type-II seesaw in $\mathrm{SO}(10)$, Nucl. Phys. Proc. Suppl. 188 (2009) 332 [INSPIRE].

[32] S. Blanchet, P.S.B. Dev and R.N. Mohapatra, Leptogenesis with TeV scale inverse seesaw in SO(10), Phys. Rev. D 82 (2010) 115025 [arXiv: 1010.1471] [inSPIRE].

[33] P. Di Bari and L. Marzola, SO(10)-inspired solution to the problem of the initial conditions in leptogenesis, Nucl. Phys. B 877 (2013) 719 [arXiv:1308.1107] [InSPIRE].

[34] P. Di Bari, L. Marzola and M.R. Fiorentin, Decrypting SO(10)-inspired leptogenesis, arXiv: 1411.5478 [INSPIRE].

[35] R.N. Mohapatra and B. Sakita, $\mathrm{SO}(2 N)$ grand unification in an $\mathrm{SU}(N)$ basis, Phys. Rev. D 21 (1980) 1062 [INSPIRE].

[36] P. Nath and R.M. Syed, Analysis of couplings with large tensor representations in $\mathrm{SO}(2 N)$ and proton decay, Phys. Lett. B 506 (2001) 68 [Erratum ibid. B 508 (2001) 216] [hep-ph/0103165] [INSPIRE].

[37] C.S. Fong, M.C. Gonzalez-Garcia, E. Nardi and E. Peinado, New ways to TeV scale leptogenesis, JHEP 08 (2013) 104 [arXiv:1305.6312] [INSPIRE].

[38] R. Barbieri, P. Creminelli, A. Strumia and N. Tetradis, Baryogenesis through leptogenesis, Nucl. Phys. B 575 (2000) 61 [hep-ph/9911315] [InSPIRE].

[39] T. Endoh, T. Morozumi and Z.-h. Xiong, Primordial lepton family asymmetries in seesaw model, Prog. Theor. Phys. 111 (2004) 123 [hep-ph/0308276] [INSPIRE].

[40] A. Abada, S. Davidson, F.-X. Josse-Michaux, M. Losada and A. Riotto, Flavor issues in leptogenesis, JCAP 04 (2006) 004 [hep-ph/0601083] [INSPIRE].

[41] E. Nardi, Y. Nir, E. Roulet and J. Racker, The importance of flavor in leptogenesis, JHEP 01 (2006) 164 [hep-ph/0601084] [INSPIRE].

[42] L. Covi, E. Roulet and F. Vissani, CP violating decays in leptogenesis scenarios, Phys. Lett. B 384 (1996) 169 [hep-ph/9605319] [INSPIRE].

[43] D. Aristizabal Sierra, L.A. Muñoz and E. Nardi, Purely flavored leptogenesis, Phys. Rev. D 80 (2009) 016007 [arXiv: 0904.3043] [InSPIRE].

[44] C.S. Fong, M.C. Gonzalez-Garcia and E. Nardi, Early universe effective theories: the soft leptogenesis and R-genesis cases, JCAP 02 (2011) 032 [arXiv:1012.1597] [INSPIRE].

[45] E. Nardi, Y. Nir, J. Racker and E. Roulet, On Higgs and sphaleron effects during the leptogenesis era, JHEP 01 (2006) 068 [hep-ph/0512052] [INSPIRE].

[46] G. Altarelli and G. Blankenburg, Different $\mathrm{SO}(10)$ paths to fermion masses and mixings, JHEP 03 (2011) 133 [arXiv: 1012.2697] [InSPIRE]. 
[47] B. Dutta, Y. Mimura and R.N. Mohapatra, Suppressing proton decay in the minimal SO(10) model, Phys. Rev. Lett. 94 (2005) 091804 [hep-ph/0412105] [INSPIRE].

[48] B. Dutta, Y. Mimura and R.N. Mohapatra, Neutrino mixing predictions of a minimal SO(10) model with suppressed proton decay, Phys. Rev. D 72 (2005) 075009 [hep-ph/0507319] [INSPIRE].

[49] S. Davidson, From weak scale observables to leptogenesis, JHEP 03 (2003) 037 [hep-ph/0302075] [INSPIRE].

[50] Planck collaboration, P.A.R. Ade et al., Planck 2013 results. XVI. Cosmological parameters, Astron. Astrophys. 571 (2014) A16 [arXiv:1303.5076] [inSPIRE].

[51] WMAP collaboration, C.L. Bennett et al., Nine-year Wilkinson Microwave Anisotropy Probe (WMAP) observations: final maps and results, Astrophys. J. Suppl. 208 (2013) 20 [arXiv:1212.5225] [INSPIRE].

[52] S. Antusch, J. Kersten, M. Lindner and M. Ratz, Neutrino mass matrix running for nondegenerate seesaw scales, Phys. Lett. B 538 (2002) 87 [hep-ph/0203233] [INSPIRE].

[53] D. Meloni, T. Ohlsson and S. Riad, Effects of intermediate scales on renormalization group running of fermion observables in an $\mathrm{SO}(10)$ model, JHEP 12 (2014) 052 [arXiv: 1409.3730] [INSPIRE]. 